\title{
Research On Optimization In NC Bending Process Of Thin-walled Tube Based on Orthogonal Experiment
}

\author{
Ren Sheng-le*, Wang Peng and Dai Ye \\ School of Mechanical \& Power Engineering, Harbin University of Science and \\ Technology, Heilongjiang Harbin 150080, China \\ *renshengle@163.com
}

\begin{abstract}
In the process of research and development of tube bending forming technology, the way of improving the forming quality and establishing the optimization of forming process parameters has become an important problem that should be solved quickly. This text simulated the process of bend forming with the way of finite element, combined with the effects of geometric and physical parameters on the forming quality of bending pipe, taking the important parameters which are measurements of the pipe wall thickness variation as test indexes, made the virtual orthogonal experiment and gained the laws of the bending angle, relative bending radius, relative wall thickness, thrust, pipe friction coefficient between mode have effects on the forming quality of wall thinning rate and wall thickening. Through the comprehensive analysis and additional test, obtained the optimal parameters values, improved the accuracy of simulation and the optimization results of orthogonal test, improved the quality of tube bending forming.
\end{abstract}

Keywords: finite element, bend, parameters, orthogonal experiment

\section{Introduction}

The NC tube bending forming technology for twenty-first Century relates to material science, mechanics, mechanical and computer application and so on. The thin-walled tube NC bending process is a complex nonlinear process which is $\mathrm{V}$ multi-parameters coupling interaction. If the forming parameters is improper selection, It is easy to make the pipe lateral thinning, cracking, inner wrinkling and the cross section deformation, resilient and other defects. These defects not only bring huge waste and not conducive to reducing production cost and increasing the production efficiency, but also reducing the size precision of bend, harming the functional performance and Seriously restrict the development of NC tube bending forming technology. So the research of $\mathrm{NC}$ tube bending process parameters optimization is very important.

This paper studies the method of cold forming of thin-walled tube NC bending mandrel. The traditional bend processing depended on the designer's experience to adjust process parameters repeatedly. The process of text is complex, the cycle is long, with occasional and cannot guarantee the results are optimal. With the development of computer technology and optimization theory, the way of change the method of tube bending quality is also increasing. But by the way of combine the finite element software with optimization method to research the bend forming quality has become a new trend. Zhang Yaowu ${ }^{[1]}$ have researched the hot forming bend process parameter which is based on the virtual orthogonal experiment, taking advantage of the finite element method making Computer simulation to the whole forming process, used the virtual orthogonal test method making optimal design to the simulation results for different parameters, acquiring the influence of the parameters on the hot forming 
process and then the optimal parameter values. Wu Xiulin ${ }^{[2]}$ through the optimization design of intelligent pipe bending machine based on Stewart platform for motion control. To satisfy the special trajectory of intelligent bending machine's work space is as far as possible and the premise motion transmission performance is as good as possible. Using the genetic algorithm in the structural parameters on the Stewart platform mechanism for multi-objective optimization design, in order to improve the dynamic performance of machine kinematics objective.

The virtual orthogonal test is a method of research and treat the multi factor experiment. With the practical experience and theoretical knowledge, using orthogonal table to arrange experiments, through a balanced sampling, it can find the best conditions of production and scientific research which is the optimal scheme ${ }^{[3-6]}$.

The text use finite element method to imitate the whole process of the bend forming using the multi index orthogonal test to optimize the design of the geometrical and physical parameters' simulation results, acquiring the influence of the parameters on the bend forming quality and then the optimal parameter values.

\section{The NC Tube Bending Simulation Experiment}

\subsection{The Bend Shaping Principle}

The common methods of bending pipe are bent push [7-8]. The thin-walled tube bending push processing schematic diagram is shown in Figure 1.

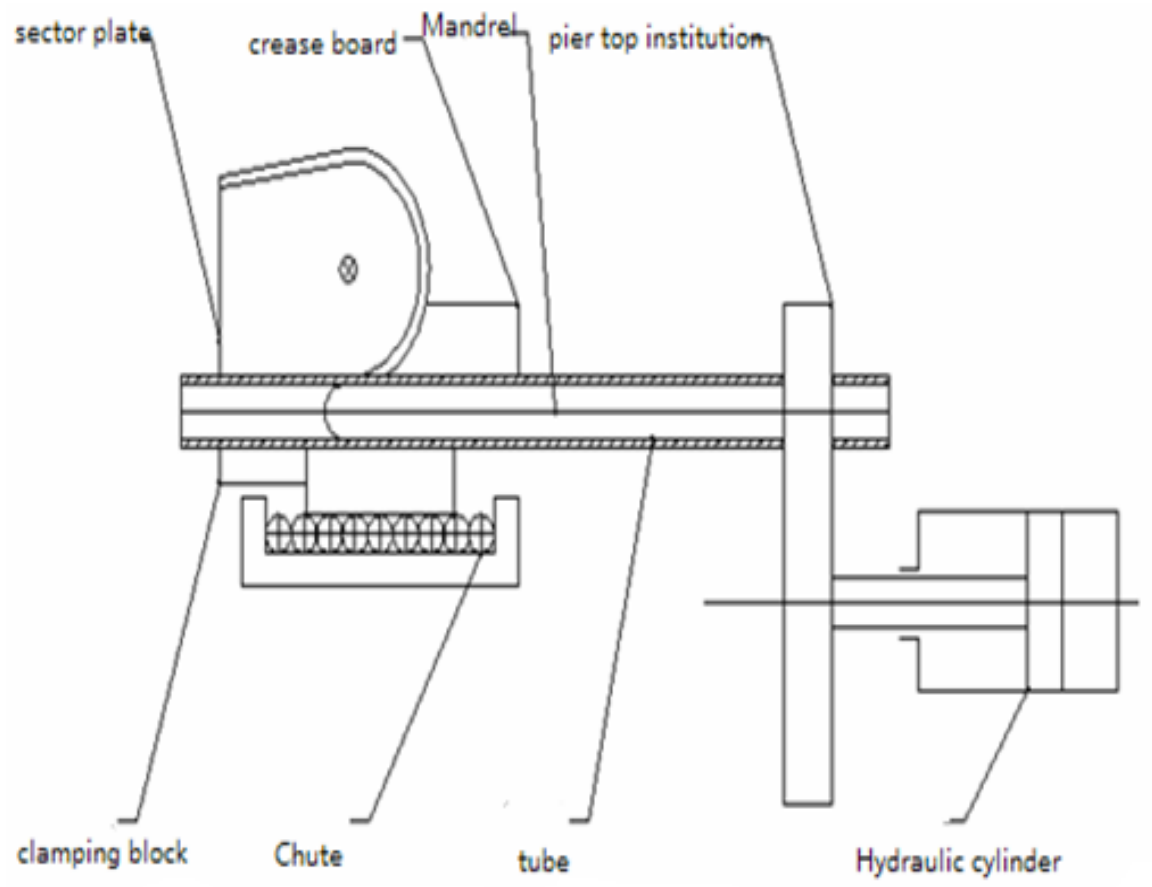

Figure 1. The Diagram of Cold-formed Pushing

The principal axis drive sector disk rotating around its axis. After the tube placed in the sector plate, through the chute placed on the right side of clamping block grip the tube using the pressure of hydraulic cylinder lock it relying on the chute pushing the tube and the groove 
tube applied to a pre-deformation itself and then use the pier top institutions clamping the tube to limit the rotation of the tube tail. Finally, the clamping block and the sector plate to rotate together, in the combined effects of the mold to realize the tube forming.

\subsection{Select the Bending Material}

The test tube material is $40 \mathrm{Cr}$ steel, Young's modulus is $2.2 \times 10^{11} \mathrm{~Pa}$, Poisson's ratio is 0.275 , destiny is $7.86 \times 10^{-3} \mathrm{~g} / \mathrm{mm}^{3}$, is one of the most commonly used tube type in actual production. The Outer diameter of tube is $108 \mathrm{~mm}$, Bending radius is $152 \mathrm{~mm}$, Wall thickness is $5 \mathrm{~mm}$.

\subsection{The Select of Finite Element Software}

MSC.MARC is an advanced nonlinear finite element software functions, has strong analysis ability structure, and can solve various nonlinear problems. And MSC.MARC has two times the development of the friendly interface. That is easy for model of mould input and data output processing. This text used the finite element method with MSC.MARC2005 software.

\subsection{The Established of Finite Element Model}

In this text, the tube model is produced by PROE and MARC to ensure the accuracy of the simulation and save calculation time, Ignoring the elastic properties of mould, simplify it as rigid constraint surface. The tube is set to the $3 \mathrm{D}$ entity, using eight node hexahedron divide three dimensional parametric grid. The Boundary condition is cancel the fixed and movable clamping blocks on the basis of the original model instead of to the boundary constraint. In this text, the contact treatment of tube model is that treat the other mould as euclidean body except the tube. In the pipe bending process can not stress and deformation and the friction properties between the mould and the tube by Kulun friction model. The friction factor: tube / crease block 0.05 , tube / bending die 0.1, tube/plug 0.15, tube/ press cake 0.2. Because of this text is research the cold bending technology, so there is no need to consider the influence of temperature on the analysis, we can use default temperature of the software. Simulating the forming process based on the condition above, the tube numerical simulation is shown in Figure 2.



Figure 2. Tube Bending Forming Numerical Simulation Model 


\subsection{Select the Forming Parameters}

The process parameter of bend is mainly include material parameter and bend geometry parameter, since the parameters increase will make the orthogonal experiment factors increase, considering each factor contains four levels, so do some scientific analysis and comparison to these parameters, selecting the parameter which have a huge influence on forming quality as the factor of orthogonal test [9]. The test will be to optimize these parameters. This paper select material parameters as design constant and select angle of bend, the relative bending radius, the relative wall thickness, thrust, pipe friction coefficients as design variables.

Parameter values should be combined with the actual production experience and previous experimental results to select the reasonable range and short-cut process as uniform as possible, so that we can analysis the parameters influencing to the forming of tube.

\section{Test Method for Parameter Optimization}

The purpose to analysis bend forming simulation result is optimize the process parameter and reveal the deformation regularity [10-12]. The bend forming quality is mainly depends on the wall thickness reduction and wall thickening of bend forming. This paper will use the wall thickening and wall thickness reduction rate as a measure of process parameters and the two test index.

\subsection{The Design Orthogonal Test}

In the experiment, treat the forming pipe wall thickening and wall thickness reduction rate as two indicators for the orthogonal test, before forming the bend's bending angle, relative bending radius, friction coefficient of relative wall thickness, thrust, tube mode is treated as orthogonal experiment factor which Is effect the tube bending forming quality. For the accurate assurance test, each factor select four level. According to the above principles, made factor level table, as shown in Table 1.

Table 1. Factor Level Table

\begin{tabular}{c|c|c|c|c|c}
\hline Factor & $\begin{array}{c}\mathrm{A} \\
\text { Bending angle } \\
{ }^{\circ}\end{array}$ & $\begin{array}{l}\mathrm{B} \\
\text { Relative } \\
\text { bending } \\
\text { radius }\end{array}$ & $\begin{array}{l}\mathrm{C} \\
\text { Relative } \\
\text { wall } \\
\text { thickness }\end{array}$ & $\begin{array}{c}\mathrm{D} \\
\text { Thrust } \\
/ \mathrm{N}\end{array}$ & $\begin{array}{c}\mathrm{E} \\
\text { The friction } \\
\text { between the pipe } \\
\text { die }\end{array}$ \\
\hline 1 & 60 & 2.19 & 0.074 & 2000 & 0.10 \\
\hline 2 & 100 & 2.41 & 0.111 & 4000 & 0.15 \\
\hline 3 & 140 & 3.33 & 0.130 & 6000 & 0.20 \\
\hline 4 & 180 & 4.26 & 0.175 & 8000 & 0.25 \\
\hline
\end{tabular}

The way to analysis orthogonal test without interaction of multiple indexes include comprehensive scoring method and integrated balance method. The key of the comprehensive evaluation method is to determine the index weight, that needs a wealth of practical experience. There is no general formula in math. In view of different forming conditions of bend, this paper selects the comprehensive balance method, five factors and four levels table $L_{16}\left(4^{5}\right)$. 
The basic steps of the integrated balance method: firstly, analysis each index, that is same to the analysis method of single index and then find the optimal production condition of each index. Finally, comprehensive balancing the optimal condition of each index and find out the consideration of each index all the best possible conditions. The orthogonal design is shown in Table 2.

Table 2. Orthogonal Test Scheme

\begin{tabular}{|c|c|c|c|c|c|c|c|}
\hline Factor & \multirow[t]{2}{*}{$\mathrm{A}$} & \multirow[t]{2}{*}{ B } & \multirow[t]{2}{*}{$\mathrm{C}$} & \multirow[t]{2}{*}{ D } & \multirow[t]{2}{*}{$\mathrm{E}$} & \multicolumn{2}{|c|}{ experimental results } \\
\hline Text & & & & & & $\begin{array}{c}\text { Wall thickening } \\
1 \%\end{array}$ & $\begin{array}{c}\text { Wall thickness } \\
\text { reduction } \%\end{array}$ \\
\hline 1 & 1 & 1 & 1 & 1 & 1 & 17.45 & 12.45 \\
\hline 2 & 1 & 2 & 2 & 2 & 2 & 14.21 & 11.75 \\
\hline 3 & 1 & 3 & 3 & 3 & 3 & 12.43 & 11.41 \\
\hline 4 & 1 & 4 & 4 & 4 & 4 & 6.21 & 5.85 \\
\hline 5 & 2 & 1 & 2 & 3 & 4 & 11.21 & 10.87 \\
\hline 6 & 2 & 2 & 1 & 4 & 3 & 11.73 & 11.33 \\
\hline 7 & 2 & 3 & 4 & 1 & 2 & 14.33 & 12.59 \\
\hline 8 & 2 & 4 & 3 & 2 & 1 & 10.91 & 9.42 \\
\hline 9 & 3 & 1 & 3 & 4 & 2 & 8.77 & 7.65 \\
\hline 10 & 3 & 2 & 4 & 3 & 1 & 8.36 & 6.47 \\
\hline 11 & 3 & 3 & 1 & 2 & 4 & 9.68 & 6.54 \\
\hline 12 & 3 & 4 & 2 & 1 & 3 & 7.83 & 7.28 \\
\hline 13 & 4 & 1 & 4 & 2 & 3 & 6.36 & 5.76 \\
\hline 14 & 4 & 2 & 3 & 1 & 4 & 6.85 & 5.71 \\
\hline 15 & 4 & 3 & 2 & 4 & 1 & 6.27 & 4.87 \\
\hline 16 & 4 & 4 & 1 & 3 & 2 & 6.87 & 4.22 \\
\hline
\end{tabular}


Table 3. The Result Analysis Table

\begin{tabular}{c|c|c|c|c|c|c|c|c|c|c}
\hline & \multicolumn{4}{|c|}{ Wall thickening } & \multicolumn{5}{c}{ Wall thickness reduction } \\
\hline$k_{1}$ & 12.58 & 10.95 & 11.43 & 11.62 & 10.75 & 10.37 & 8.99 & 8.64 & 9.51 & 8.30 \\
\hline$k_{2}$ & 12.05 & 10.29 & 9.88 & 10.29 & 11.05 & 11.05 & 8.82 & 8.69 & 8.37 & 9.05 \\
\hline$k_{3}$ & 8.66 & 10.68 & 9.74 & 9.72 & 9.59 & 6.99 & 8.85 & 8.55 & 8.24 & 8.95 \\
\hline$k_{4}$ & 6.59 & 7.96 & 8.82 & 8.25 & 8.49 & 5.14 & 6.69 & 7.67 & 7.43 & 7.24 \\
\hline$R$ & 5.99 & 2.99 & 2.61 & 3.37 & 2.56 & 5.91 & 2.30 & 1.02 & 2.08 & 1.81 \\
\hline
\end{tabular}

The results is shown in Table 3, In the text of No.4,the Wall thickening is lowest, the Level is $A_{1} B_{4} C_{4} D_{4} E_{4}$, but in the text of No.16 the Wall thickness reduction is the lowest, the Levelis $A_{4} B_{4} C_{1} D_{3} E_{2}$. The relationship between primary and secondary of five factor to two index: Wall thickening $(A>D>B>C>E)$, Wall thickness reduction $(A>B>D>E>C)$. Therefore, the bending angle is the main factor that affects the quality of the tube forming, so we should choose small bend angle when design pipe geometry parameters. As shown in Figure 3, 4 show the index map.
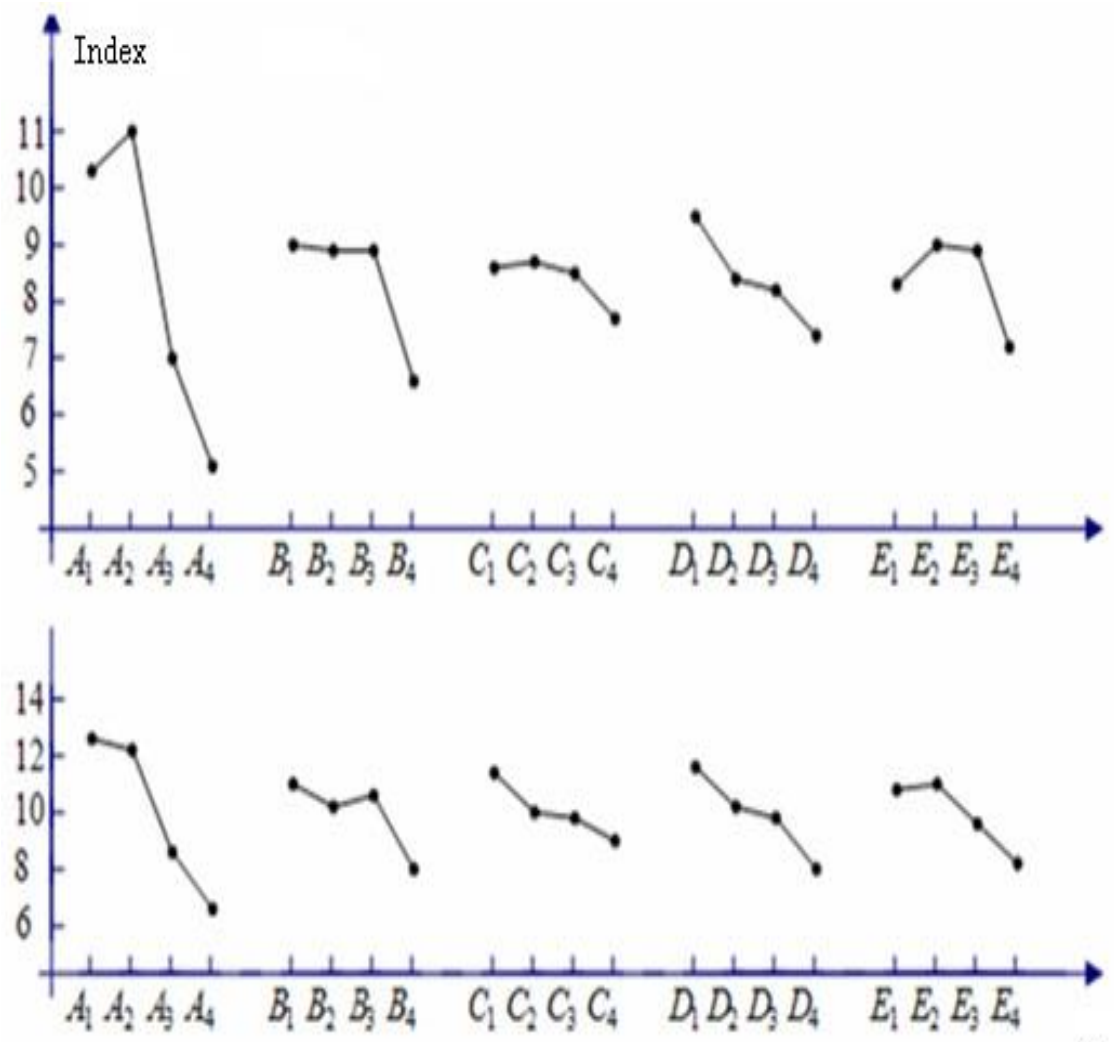

Factor

Figure 3. Factor-index Chart 
Integrated survey the two index, the higher the rate of wall thickening, the bending forming quality is worse. From Figure 3 and the primary and secondary factors know that the effect of $\mathrm{A}$ is the most important, only choose $180^{\circ}$ is suitable. The factor B have same influence trend to the two index, but the effects on wall thickening rate is greater than the external wall thinning rate so select 2.19 is the most appropriate. The influence of factor $\mathrm{C}$ is small to the two index. But for the same diameter bend, With the relative wall thickness increase, the thinning rate and thickening rate become smaller, so choose 0.111 . The influence of factor $\mathrm{D}$ is important to bend forming quality, with the increase of thrust, forming quality is better, then, with the increased boost, two indicators have stabilized, so choose $3000 \mathrm{~N}$. Factor E are the secondary factor, but it has more influence to Wall thickness reduction than Wall thickening, so choose 0.15 .

The scheme did not in the 16 group test that have been done, so we must do more test, getting the wall thickening rate was $6.15 \%$ and the outer wall thinning rate was $4.57 \%$, but in the orthogonal test, the lowest inner wall thickening and wall thinning rate is at group 4 and group 16, the additional test significantly better. Verification test proved that the group which use the method of general equilibrium analysis is the best group.

\section{Experimental Verification}

It is the optimization results of this test that bending Angle is $180^{\circ}$, the relative bending radius is 2.19 , relative wall thickness is 0.111 , thrust is $3000 \mathrm{~N}$, the friction coefficient between the pipe die is 0.15.The flaw from processing the fitting surface with these technological parameters are smooth, without fold, tear and so on. The inside of the axial wall thickness simulation compared with the experimental results are shown in figure 4.The experimental pipe wall thickness distribution is identical with the simulation results. Distribution is in good condition.

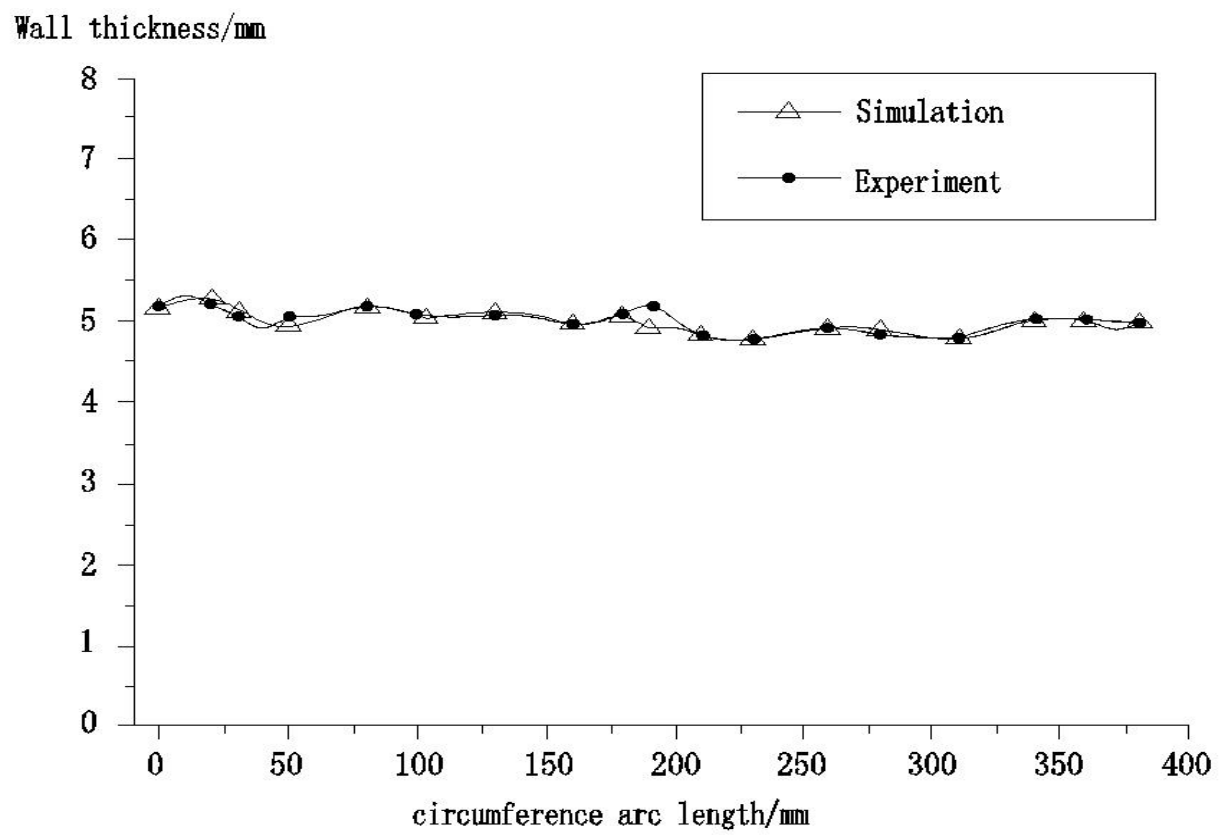

Figure 4. The Comparison of Simulative and Experimental Results 


\section{Conclusion}

These experiments have theoretical guidance for choose and design of the tube geometric parameter. In the pressure condition, the more smaller of bend deformation, the more safe it is, adopting uniform thickness, little angle of bend and little bend radius is the safety design idea. But in the actual production, we should overall consideration the effect of various parameter to the forming quality, then get the optimal production. Especially the cold bending production, the thinning rate play a important role in bending performance, the smaller the bend radius the bigger the thinning rate. In another word, the pipe wall thickness thinning is more obvious. The wall thickness thinning is harmful to pipe bending mechanical properties, in the same pressure condition, the pipe thinning part will not withstand pressure and elastic deformation. To sum up, get the following conclusion:

1) By orthogonal experimental design theory as the foundation, selecting the bending angle, relative bending radius, the relative wall thickness, thrust, the coefficient of friction as the experimental factors and study on the influence of various factors on the wall thickness change rate.

2) Using the integrated balance method to select the optimal combination of parameters and verifying the rationality of the optimal parameter combination through additional experiments.

3) The optimized process parameter is used for processing, the bend forming quality is good, and verify the optimization process accurately and efficiently.

\section{Acknowledgements}

The research is supported by National Natural Science Foundation Project (51105120), Post-doctoral funding projects in Heilongjiang province (LBH-210115).

\section{References}

[1] Y. Zhang, W. Ceng and Y. Zhou, "Optimal design of technological parameters for hot-pushing pipe-bending based on virtual orthogonal experiment", Journal of Plasticity Engineering, vol. 16, no. 6, (2009), pp. 91-95.

[2] X. Wu and L. Li, "The Optimal Design and Kinematic Control of Parallel Machine Tool for Bending Metal Tools Based on Stewart Platform", Xi'an Jiao Tong University, (2008).

[3] J. Fan, "The theoretical analysis of bending parameters in the forming of tube", science and technology information, vol. 2, (2013), p. 133.

[4] T. Welo, F. Paulsen and T. J. Brobak, "The behavior of thin walled aluminum alloy profiles in rotary draw bending-a comparison between numerical and experimental results", Journal of Materials Processing Technology, vol. 45, (2008), pp. 173 180.

[5] M. Elchalakani, XL. Zhao and RH. Grzebieta, "Plastic mechanism analysis of circular tubes under pure bending”, International journal of Mechanic Science, vol. 44, (2009), pp. 117 1143.

[6] J. Luo, "Determination of the Influence of the Bends Geometric Parameter of the Amount of Deformation with an Orthogonal Testing Method [J]", pipeline technology and equipment, vol. 4, (2010), pp. 10 12.

[7] L. Jiang, "Parameter Research and Actual Application of Large NC System Pipe Bender [J]", Bolier manufacturing, vol. 3, (2012), pp. 96-101

[8] Z. Wu, S. Liu and W. Liu, "Optimization design of sheet L-bending with method based on orthogonal experiment [J]", Journal of Lanzhou University of Technology, vol. 1, (2010), pp. 45-48.

[9] A. G. Korba, M. M. Megahed, H. F. Abdalla and M. M. Nassar, "Shakedown analysis of 90-degree mitred pipe bends", European Journal of Mechanics - A/Solids, vol. 7, no. 40, (2013), pp. 158-165.

[10] H. Li, J. Wood, R. McCormack and R. Hamilton, "Numerical simulation of ratcheting and fatigue behaviour of mitred pipe bends under in-plane bending and internal pressure", International Journal of Pressure Vessels and Piping, vol. 1, no. 101, (2013), pp. 154-160.

[11] W. Zhang, Z. He and J. Zhang, "Based on PLC Pipe Bender Control System Design [J]", Coal mine machinery, vol. 1, (2013), pp. 23-25. 
[12] C. Guofu, “Analysis on Reasons Caused by Bend Abnormal Wrinkle [J]”, Welded pipe, vol. 11, (2012), pp. 67-70. 
International Journal of Hybrid Information Technology Vol.8, No.1 (2015) 